\title{
Managing Performance to Align the Participants of Collaborative Networks: Case Studies Results
}

\author{
Roberto da Piedade Francisco, Américo Azevedo, and João Bastos \\ INESC Porto \& Faculdade de Engenharia da Universidade do Porto, \\ Rua Doutor Roberto Frias S/N \\ 4200-465 Porto, Portugal \\ \{roberto.piedade, ala, joao.bastos\}@fe.up.pt
}

\begin{abstract}
The paper underlines the interest of using a performance management system applied to monitor the strategy effectiveness and the interoperation's performance in the collaborative networks $(\mathrm{CN})$. The aim with this study is to verify if a performance management system helps decision-makers reach the alignment among participants in a collaborative network. It is expected that this work provides an adequate tool for supporting quick decisions to meet the feasible and desirable improvements in inter-organisational processes. A framework (CNPMS) is also presented, having been developed to support the dynamic performance management, which was applied in case studies of two Brazilian collaborative networks.
\end{abstract}

Keywords: Collaborative Networks, Alignment, Performance Management.

\section{Introduction}

Nowadays organisations recognize that a tight interaction and coordination among participants of their business chain, or emergent opportunities, motivates them to act in Collaborative Networks $(\mathrm{CN})$, and furthermore it is a crucial strategy to reach continuous competitiveness and to reduce business uncertainty. Therefore, this new model involves the interoperation of different organisational systems that must be integrated and orchestrated in order to provide effectiveness, bringing a suitable performance. Then, this new vision requires technology and infrastructure support, management tools and appropriate performance measurement solutions that can ensure the alignment expected regarding the strategic objectives pertaining to business among the participants in a business networked environment.

Then, this paper intends to highlight some aspects regarding performance management seeking to achieve the alignment of participants in collaborative networks. Thus, this document is organized as follows: the second section presents definitions and explanations on alignment in CNs. In the third section, an overview of the performance management is provided. Then, the fourth section addresses the results of some case studies, including the explanation of the Collaborative Network Performance Management System (CNPMS); finally, some conclusions are presented in fifth section. 


\section{Alignment in Collaborative Networks}

Collaborative networks are the interaction among entities geographically separated with distinct competencies, and sometimes distinct interests, that intend to cooperate in order to reach common goals, symbiotically in the same breeding environment [1]. This new paradigm emerged as a powerful mechanism to achieve competitiveness, excellence and agility in the turbulent market conditions. To define "collaborative network", Camarinha-Matos \& Afsarmanesh [2] argue that this term is often confused with cooperation and thus state there are other types of interactions between firms, such as coordination and networking. These interactions are defined as:

- Networking. It constitutes the interactions of communication and exchange of information between groups of organizations for their own use. This can occur even if there are no common goals among the participants.

- Coordinated Networking. Keeping premise that there are no specific common goals, the coordination of information sharing may involve the alignment of activities so that it is possible to achieve more effective results.

- Cooperation. The division of specific activities between participants. Exchange of information and redirection of activities occurs as in previous settings, but it includes the sharing of resources to achieve feasible and desirable goals.

- Collaboration. It occurs when the partners work together to plan, implement and evaluate inter-organizational processes providing the grounds for share information and resources to achieve common goals and to strengthen the each one's capacities. Acting under a single identity, it that risks, resources and responsibilities are shared, so that the benefits can be achieved.

Therefore, an interaction can occur in a simple cooperation between organizations or to advance to a level where there is coordination of inter-organizational processes that exploit information and communication among them. On the other hand, there can be cooperation where responsibilities on the inter-organizational processes and respective resources are shared, or cooperation that can advance to an even higher level with an agreement on the common strategic objectives, i.e., collaboration.

In this context, Saiz, Rodríguez \& Bas [3] argue that companies operating in CNs can maximize the participants' capabilities, once combined to achieve the strategic objectives to meet customer needs for integrated and efficient solutions. So, the alignment is very important in formulating the strategy mainly on the adjustment of systems and key processes, as well as the decision-making system [5]. Although it has other connotations, the term "alignment" can be defined as a proper or desirable coordination or relation of components [4]. However, in the management scope, it can be properly considered as a situation where strategies, organizational structures, stakeholders, stockholders, and whatever participant or process in a business environment are strictly combined under a set of roles to reach specific objectives and goals. Align the main activities of the organization around a generic strategy improves communication with customers, employees and shareholders, considering the business environment, strategies and resources established.

The alignment can be referred to as taking the following perspectives into consideration: strategic alignment, organizational alignment, operational alignment and team alignment. However, the main focus of this work does not concern about the alignment of teams or about the operational alignment. Instead, it focuses on achieving strategic 
alignment in a collaborative network supported by the organizational alignment. In this context, some factors should be considered in the organizational structure to bring organizational alignment, such as: project size, duration, the organization's experience in managing projects, the philosophy of the company's upper management regarding project management, the physical location of the project, available resources, and specific project aspects [6].

We argue that there are three moments of decision-making that can affect more significantly the strategic alignment, such as: partner selection, agreement and performance management. To achieve the alignment, these moments should be properly planned during the CN's implementation. Moreover, there are different fitting levels according to each moment of the $\mathrm{CN}$ life-cycle, which must be addressed since the selection of partners, in the agreement, and until the start-up (figure 1). After that, due to results not yet known whether that will happen during the runtime, it is expected that the measurement and performance management system helps improve the alignment. If the performance is instantiated, the decision-makers can improve the interorganisational processes and the customer solutions. At this moment, the strategic alignment should be achieved even if there are some setbacks.

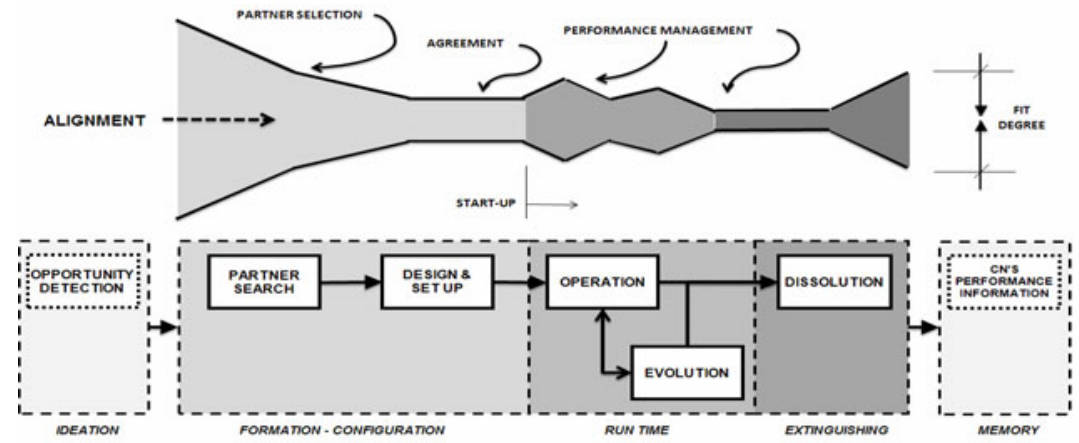

Fig. 1. Alignment through the $\mathrm{CN}$ life-cycle

Then, the alignment requires a measure, even if it is subjective, to instantiate the degree of alignment (fit degree) that occurs in the three moments mentioned above. The proposal is to use five degrees of alignment, such as: highest, $\underline{\text { high, }} \underline{\text { medium, low }}$ and lowest. This evaluation process can be obtained through fieldwork in the $\mathrm{CN}$, application of questionnaires to the participants or/and stakeholders, collaborative meetings for the review of inter-organizational processes where procedures are established for continuous improvement, and also results from the view of the board.

However, another approach is being developed to improve this alignment evaluation form in order to fill possible gaps originated from the personal and subjective evaluations. It is based on Fuzzy Logic and intends to use the result of strategic key performance indicators (KPI) set by decision-makers and turn them into qualitative assessments of compliance with the targets. Thus, through the rankings of each participant make possible to obtain the "fit degree" of the $\mathrm{CN}$ as a whole. If the Fit Degree measure is considered by participants as an important factor, both in $\mathrm{CN}$ configuration and in subsequent improvements, this may mean that, in these decision-making moments, it is possible has a CN's management processes more effective. 


\section{Managing Performance}

In a collaborative network, the decision-makers seek to improve the performance of inter-organisational processes in order to fulfill the CN's purposes. They must use systems of performance management and measurement derived from its strategies and capabilities [7]. The main principle is to integrate the goals with strategies and thus reach a better definition of the processes, their performance and their relationship with the internal and external clients.

In fact, in order to improve the management of collaborative networks, a dynamic performance management system (PMS) should be considered in order to build an interactive and aligned framework. This should support the collaborative networks in a simple and consistent manner to support decisions based on performance.

For organisations to manage this new model effectively, it is important to understand how to implement effective systems that can integrate and align interoperational performance. The use of a PMS helps organisations provide instances of performance, contribute to decision-making, and lead to the alignment of all participants in the defined strategic direction [8]. For an organization to make an effective use of the performance measurement outcomes, it should be able to make the transition from measurement to management. According to Evans, Roth \& Sturm [8], a performance measurement system is considered a subset of a PMS due the evolution of the concepts. Also, Busi \& Bititci [9] contend that measuring performance is only one practical and technical support to performance management, which is a broader approach to treat performance and its implications.

In this context, Amaratunga \& Baldry [10] state that performance management is using information from performance measurement to trigger positive changes in the systems and processes of the organization, as well as in the organizational culture, and to define objectives and goals, allocating and prioritizing features.

Nowadays, there is some specific performance management systems used in CNs, each one consisting of gathering some concepts in order to build a framework that can support decision-makers so that they can achieve the specific purposes or strategies, and identify new challenges.

The strategic alignment helps provide consistency and efficiency to the interrelationships among the CN's participants. However, to achieve organizational alignment, each one of them, must provide contributions (operation efficiency) to the CN. Then, it becomes desirable to achieve sustainable gains from the synergy conquered in collaborative processes. This can occur if a performance management system, acting as a tool, can transform the strategies into action through the alignment once happening in the relevant and critical activities [1].

Nevertheless, defining an appropriate set of categories of performance measures that meet the requirements of a particular network represents a critical step towards the establishment of an effective PMS. So, the question is "how can a performance management system make it possible to reach, or support, the strategic alignment in the CN?" The proposal is to manage performance through a collaborative network performance management system (CNPMS) that strictly depends on the alignment degree occurring in the specific moments of the $\mathrm{CN}$ life-cycle. Therefore, it is necessary to know how to define, configure and implement an effective CNPMS in order to support the $\mathrm{CN}$ decision-makers. 


\section{Results of Case Studies}

The exploratory case studies reviewed in this paper deal with the practical application of the CNPMS framework [11], and its support methodologies, considering the propositions of Afsarmanesh, Marik \& Camarinha-Matos [12] to classify the network topology. In Case 1, a temporary partnership established to explore short-term market opportunities is addressed, and, in Case 2, a dynamic project-based partnership without a dominant partner is addressed.

\subsection{Approaches and Methodologies}

The framework CNPMS proposed by Azevedo \& Francisco [11] was developed using the Case Study Research (CSR) in order to drive the research method and the Soft System Methodology (SSM) to set and reset performance indicators [13]. It is based on two main layers: data and information, and functionality. The first one concerns the data acquisition and management repository, which intends to provide reliable information based on trust, competences (skill level), experience and know-how through the historical performance of the participants. Thus, it is possible to save time in the Partner Search phase (Figure 2).

The second layer consists of three performance functionalities, such as 1) network performance that supports the $\mathrm{CN}$ phases Design \& Set-up and Evolution, using performance benchmarking, and outlines the performance targets that will be adapted and improved during processes improvement; 2) real-time performance to instantiate the inter-organisational performance. In the Operation phase, the static and dynamic indicators applied in real time in order to solve emerging problems and to make improvements (Evolution phase) are validated; and 3) performance evaluation in order to understand in the Dissolution phase, whether the $\mathrm{CN}$ has achieved its objectives and set the memory performance, and has also learned from the analysis itself by creating a repository of information about the performance generated by participants at each stage of the life-cycle.

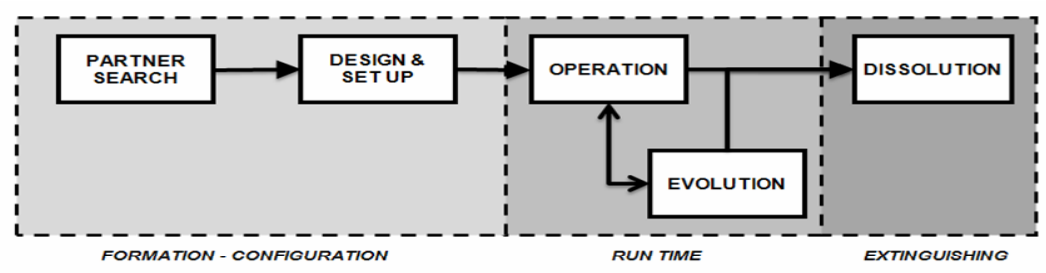

Fig. 2. Collaborative network life-cycle

The referred framework intends to motivate organizations to monitor the strategy effectiveness and the interoperation's performance of collaborative networks, i.e., to continuously monitor the intra- and inter-organisational performance. Therefore, some steps must be followed so that it is possible to manage the CNPMS, such as: defining strategies and inter-operations, and performance management skills; undertaking effective analysis about the benefits for each participant; choosing a set of 
indicators for the CNPMS; checking alignment (fit degree) in the specified moments; monitoring the performance targets and checking whether it fits with those originally proposed; reconfiguring processes of inter-operations in the event of poor performance; and checking if the previous intentions are still valid.

\subsection{Case Studies}

Some case studies were observed as part of a $\mathrm{PhD}$ program, and thus partial results obtained during the practical application of the model CNPMS [11] in some Brazilian collaborative networks are presented as follow.

Case Study 1. LogVale Logistics, a small company that provides logistics operations, seeking solutions to reduce the client's business cost. Initially, in the year 2007, a cooperation project was formed with a motorcycle dealership and both agreed to consider a collaborative network acting as a temporary partnership that would be established in order to explore market opportunities on the short term [12]. Soon after, they included three more business management consulting and ICT services firms.

On the strategic planning of this $\mathrm{CN}$, the basic issues to consider had to do with trust and management of inter-operational processes. In the beginning, the application of the CNPMS model was not so easy because there were some difficulties concerning computer systems interoperability, different management backgrounds, low collaborative partnerships experience, and so on.

Then, the alignment measurement (table 1) helped the decision-makers to implement actions towards to better manage the inter-operational processes. At the partner selection moment, the alignment was interpreted only by the two initial participants. When this occurs, the first goal that is to know the participants' skills were compromised because there was a lost opportunity to improve the alignment of the other participants to the main objectives and goals. In the following moment, the agreement, reviewed inter-operational procedures were presented and the goals properly defined inducing the partners, once questioned, to conclude that they were highly aligned. Therefore, during the run time, many meetings were carried out to measure strategic alignment considering that defined KPIs were provided trustworthy information that allows decision-making improvements.

Table 1. LogVale Logistics case results

\begin{tabular}{lll}
\hline Decision-making moments & Form & Fit Degree \\
\hline Partner Selection & Board view & High \\
Agreement & Questionnarie & High \\
Performance management & Critical analysis & Medium \\
\hline
\end{tabular}

The Fit Degree measurement can properly induce, at least, the visualization of processes or/and participants with poor performance allowing then correct timely wrong actions.

In 2008, the $\mathrm{CN}$ became extinct because the initial intentions were no longer the same, although the initial partners continued their trade. When the contributions of the partners responsible for consulting and ICT are integrated in the inter-operations, they no longer have an active participation in the project. 
Case Study 2. Pet shop retailers network, a small Brazilian collaborative network that congregates participants interested in achieving benefits through the joint formulation of strategies and operations in a regional market. This $\mathrm{CN}$ was created within the Empreender Project, sponsored by the Brazilian Support Service to Micro and Small Enterprises (SEBRAE). It is an initiative to increase entrepreneurship actions of the micro and small companies so that they can benefit from the synergy among competitors within the same branch business. It seeks to address common problems with regard to common suppliers, logistics services, marketing, asset security, employee training, among others, which can be contracted through aligned strategies. It intends to reduce costs, increase bargaining power with suppliers. In conclusion, they work together towards an increase in competitiveness.

This collaboration group includes about twenty participants. This group acts as a dynamic project-based partnership without one dominant participant [12]. SEBRAE has expertise in business management and provides resources to support planning activities, consulting in marketing issues, among others, pointing the way forward.

Then (table 2), in the partner selection moment, only the roles imposed by the Executive Direction of the Commercial and Industrial Association of the Region Garavelo (ACIRG), an association that congregates and encourages small retailers of this region to collaborate, were take in account. So, when asked about the alignment on the goals to pursue, the participants had significant differences in terms of the concepts and competencies appearing three different groups with distinct concerns. Once questioned, the partners concluded that they were not aligned with the strategies proposed. In the following moment, the agreement, the reviewed strategic planning was presented in many sessions by consultants promoting with this action the balance of different proposals and narrowed the alignment on the strategic objectives. It was noted that problems related to trust have been solved and, once questioned, partners concluded that they were satisfactorily aligned with the new goals. Furthermore, during the run time many activities were jointly carried out by the participants, such as: marketing campaigns, purchases, training sessions, outsourced services, among others. Then, in an evaluation meeting, the participants concluded that there was a medium alignment degree and they would need for more time to reach alignment because due the different perceptions, or specificities.

Table 2. Pet shop retailers case results

\begin{tabular}{lll}
\hline Decision-making moments & Form & Fit Degree \\
\hline Partner Selection & Questionnarie & Low \\
Agreement & 2 Questionnaires & High \\
Performance management & Critical analysis & Medium \\
\hline
\end{tabular}

Measuring the Degree Fit induced at least that the perception that significant differences among participants can disrupt the necessary alignment to improve effectiveness in the inter-organisational processes.

The network continues to exist today, but still more external support is required to improve the alignment because many partners still have a deficit in knowledge management. However, individual performance has improved and there was an increase in the business experience of each participant. 


\section{Conclusions}

In fact, performance management systems are yet an important management decision support tool that represents a crucial requirement to assure effective interorganisational processes. Thus, the collaborative network $(\mathrm{CN})$ may decide on the performance results of these processes. Nevertheless, the results of the alignment measurements are important to relay the concept to participants, highlighting the need for alignment along the life-cycle of the $\mathrm{CN}$.

The performance management system must support quick decisions for the changes required in the CN's strategy, and also for possible and feasible improvements in the interoperable processes.

This approach believes that performance management is a tool to align the participants of CNs once this condition is detected by the partners involved.

\section{References}

1. Chituc, C.M., Azevedo, A.L.: Multi-Perspective Challenges on Collaborative Networks Business Environment. In: Camarinha-Matos, L.M., Afsarmanesh, H. (eds.) Collaborative Networks and their Breeding Environments, pp. 25-32. Springer, Boston (2005)

2. Camarinha-Matos, L.M., Afsarmanesh, H. (eds.): Collaborative Networks: Reference Modeling. Springer, New York (2008)

3. Saiz, J.J.A., Rodríguez, R.R., Bas, A.O.: A Performance Measurement System for Virtual and Extended Enterprises. In: Camarinha-Matos, L.M., Afsarmanesh, H. (eds.) Collaborative Networks and their Breeding Environments, pp. 285-292. Springer, Boston (2005)

4. Thefreedictionary.com, http: / / www . thefreedictionary.com/alignment (10/02/2010)

5. Kathuria, R., Joshi, M.P., Porth, S.J.: Organizational Alignment and Performance: Past, Present and Future. Management Decision. Emerald 45(3), 504-517 (2007)

6. Shimizu, T., Carvalho, M.M., Laurindo, F.J.B.: Strategic Alignment Process and Decision Support Systems: Theory and Case Studies. IRM, Hershey (2006)

7. Kaplan, R.S., Norton, D.P.: The Balanced Scorecard - Measures that Drive Performance. Harvard Business Review, 71-79 (January-February 1992)

8. Evans, S., Roth, N., Sturm, F.: Performance Measurement and Added Value of Networks. In: A Research Agenda for Emerging Business Models. Kluwer, Norwel (2004)

9. Busi, M., Bititci, U.S.: Collaborative Performance Management: Present Gaps and Future Research. International Journal of Productivity and Performance Management 55(1), 7-25 (2006)

10. Amaratunga, D., Baldry, D.: Moving from performance measurement to performance management. Facilities. Emerald 20(5-6) (2002)

11. Azevedo, A.L., Francisco, R.P.: Dynamic Performance Management in Business Networks Environment. In: Cunha, P.F., Maropoulos, P.G. (eds.) Digital Enterprises Technology, pp. 401-408. Springer, New York (2007)

12. Afsarmanesh, H., Marik, V., Camarinha-Matos, L.M.: Challenges of Collaborative Networks in Europe. In: Camarinha-Matos, L.M., Afsarmanesh, H. (eds.) A Research Agenda for Emerging Business Models. Springer, New York (2004)

13. Francisco, R.P., Azevedo, A.: An SSM-Based Approach to Implement a Dynamic Performance Management System. In: Camarinha-Matos, L.M., Paraskakis, I., Afsarmanesh, H. (eds.) Leveraging Knowledge for Innovation in Collaborative Networks. Springer, Berlin (2009) 